Available online on 15.01.2020 at http://jddtonline.info
Open Access to Pharmaceutical and Medical Research
unrestricted non-commercial use, provided the original work is properly cited

Open ${ }_{\text {Access }}$

Review Article

\title{
Advanced technologies in the modern era for augmented patient health care and drug delivery
}

\author{
Dev Asish*, Khanra Soma, Shah Nilay \\ Assistant Professor, Department of Pharmaceutics, Oriental College of Pharmacy. University of Mumbai, Maharashtra, India
}

\begin{abstract}
The objective of the work is to recognize the recent advancements in the modern health care and drug delivery systems. The article describes few recent developments in technology like artificial intelligence, personalized medicines, customized medicines, 3D printing, bioelectronic devices and tele pharmacy, which have the potential to augment health care and drug delivery in coming times. Personalized medication ensures precise health care as per the individual genetic makeup of the patients. The 3D printing technology enables to deliver tailor made solutions to fulfil individual patient requirements. Bioelectronic medicines and devices are new technology where the patient wears a device and its electrical signal cures certain ailments. Tele pharmacy ensures that the technological advances of telecommunications are also passed on to the patient health care sector. Moreover, it can be said that all these modern developments ensure that the quality of life improves and there comes a better control on the health care costs.
\end{abstract}

Keywords: artificial intelligence, personalized medicines, customized medicines, 3D printing, bioelectronic devices and tele pharmacy

Article Info: Received 07 Oct 2019; Review Completed 09 Dec 2019; $\quad$ Accepted 17 Dec 2019; Available online 15 Jan 2020

\section{Cite this article as:}

Dev A, Khanra S, Shah N, Advanced technologies in the modern era for augmented patient health care and drug delivery, Journal of Drug Delivery and Therapeutics. 2020; 10(1):147-152 http://dx.doi.org/10.22270/jddt.v10i1.3838

*Address for Correspondence:

Dev Asish, Assistant Professor, Department of Pharmaceutics, Oriental College of Pharmacy. University of Mumbai,

Maharashtra, India

\section{Introduction}

As technology develops and knowledge of various fields increase, then the quality of life should also improve. Ironically modern times has brought many new diseases and dreaded ailments also. But the logical and rational use of scientific knowledge can surely be helpful in overcoming and finding cure to many diseases. Few technology advancements like artificial intelligence, personalized medicines, customized medicines, 3D printing, bioelectronic devices and tele pharmacy. The current method of diagnosis and treatment of diseases can be said to be kind of "one pill fits all" approach. The underlying problem of such an approach is that all patients do not react in the similar fashion to the same line treatment. Hence there arises a need to identify the specific cause which is the root cause of difference in reaction to same medicaments. The future of medical treatments will be oriented towards shifting the focus to personalized medications. The comparison of conventional treatment and personalized treatment is depicted in Figure 1.

Artificial Intelligence is the branch of computer science which aids in problem solving by help of symbolic programming. Over the recent years it has evolved hugely into a science that has applications in health care, engineering and business arena. Artificial intelligence was born in 1956. [1] The use of various modern technologies in pharmacy profession to be potential for growth in this sector is unavoidable. The usage of artificial intelligence in discovering the drug is having an edge over traditional methods due to the ability that it targets the proteins whose structure is not well defined. The main aim of this development in pharmacy is to generate systems that can function intelligently and independently, and can evaluate human perception, speech recognition, decision making and conversions between languages in the analysis of complicated medical data by using composite algorithms and software. [2] The scope for use of automation and robotics in a range of pharmaceuticals, chemical analysis and the developments in dissolution testing and the analytics processing of metered-dose inhalers are immense. Although the level of judgement and expertise that pharmacists provide can't be replaced by robots, but they are taking routine tasks such as counting pills, spot and gas welding, sealing assembly and handling various tools. It not only rescues the time and cost that is needed for drug development but also make the pharmacy profession more efficient. [3] [4] 


\section{D Printing}

3D Printing technology has the potential to have a great positive impact on the medical devices industry and pharmaceutical industry. Although this technology has been there since long time, only recently it has caught the attention of public and the USFDA has already given approval of 3D tablets and other medical devices. The process of this technique which is also known as additive manufacturing based on advanced technologies processed in pharmaceuticals is to make 3D solid objects from a digitalized file. It is $2 \mathrm{D}$ printing-several many times, where the printer heap layers to originate parts, the prospective impact of 3D printing have shaken up many clinical trials and it is easier to upgrade single dosage form unit based on patient's height, weight and had a great impact on requirements of all drugs for therapy.[5]

\section{Current Medicine \\ One Treatment Fits All}

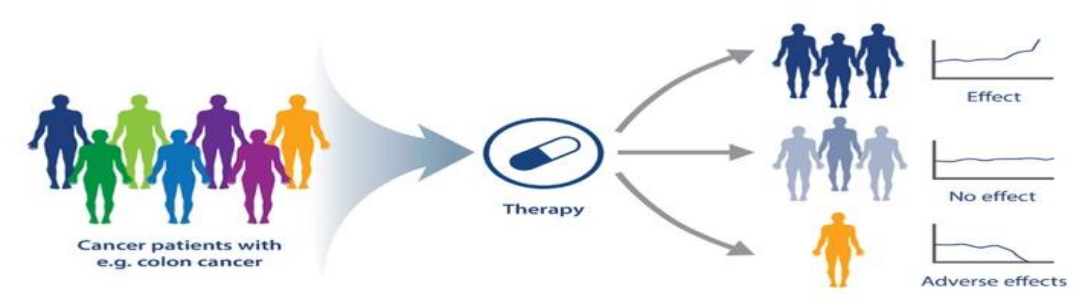

Future Medicine

More Personalized Diagnostics
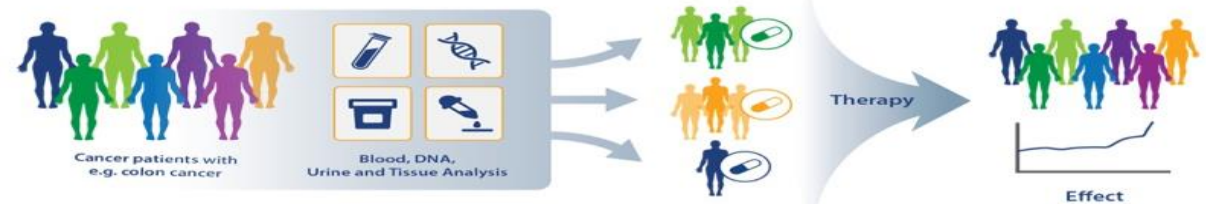

Figure 1: Conventional medication and personalized medications

The quantity of scientific papers recorded in Web of Science Core Collection containing a term B3D printing^ or B3D printed $^{\wedge}$ in the title increased from 59 in 2012 to 1573 in 2017. Moreover, the number of citations of these papers has drastic change in growing from 209 to 12,411 within same period. Narrowing the searching results to the category of pharmacy/pharmacology gives no result in 2012, however 77 records were found up to 2017, which also shows a great interest in the 3DP methods in pharmaceutical sciences.[6]
Fused deposition modeling (FDM), a type of 3D printing technology, is the most estimated in production of dosages forms because of the printers; printing precision, fundamental to guaranteeing medicine quality parameters; and hot-melt extrusion are very inexpensive. The main methods are based on powder solidification, liquid solidification and extrusion. [7]

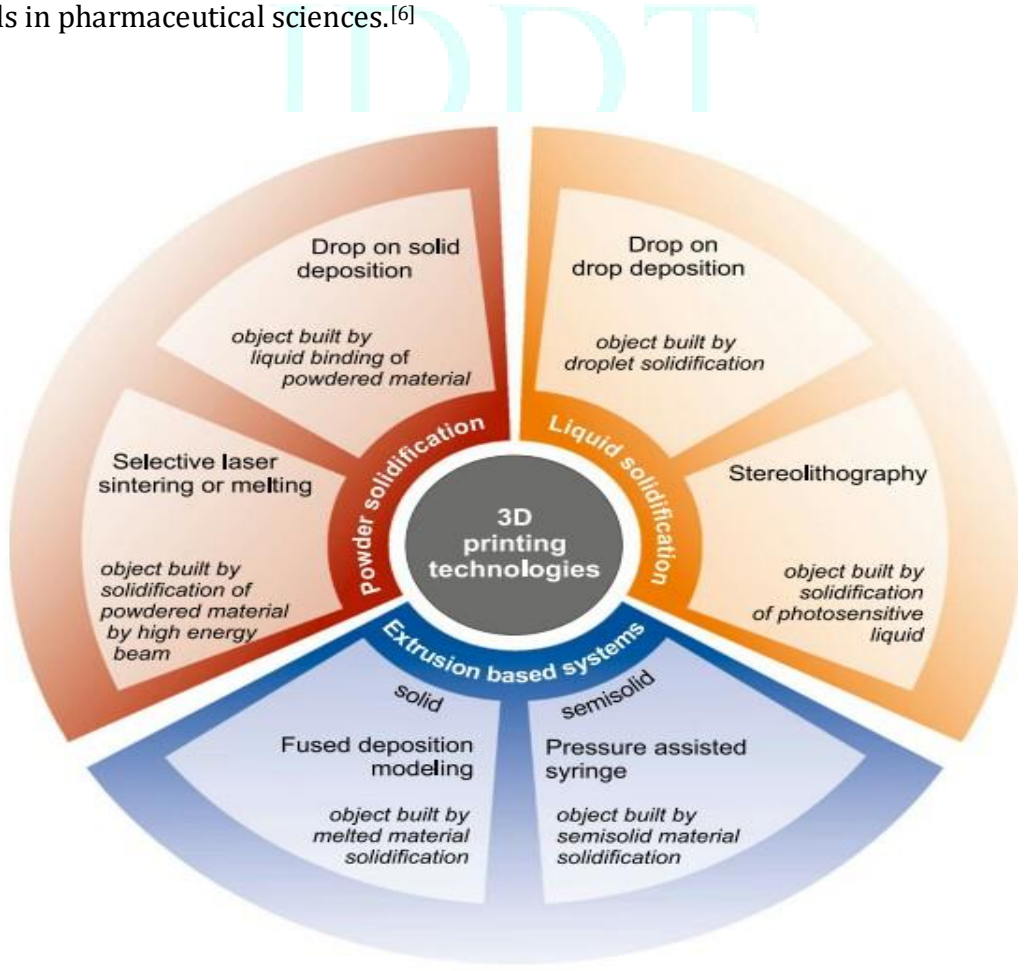

Figure 2: The progress of 3D printing technologies is explained in above diagram. 
The 3D printing technique gained an importance in the pharmaceutical and medical applications due to the possibility of rapid preparation of tailor-made objects which could be applied in personalized therapy or medicine. It is still a requirement to do investigation of possible ways with latent to generate the selected release drug delivery systems in freeform structures.

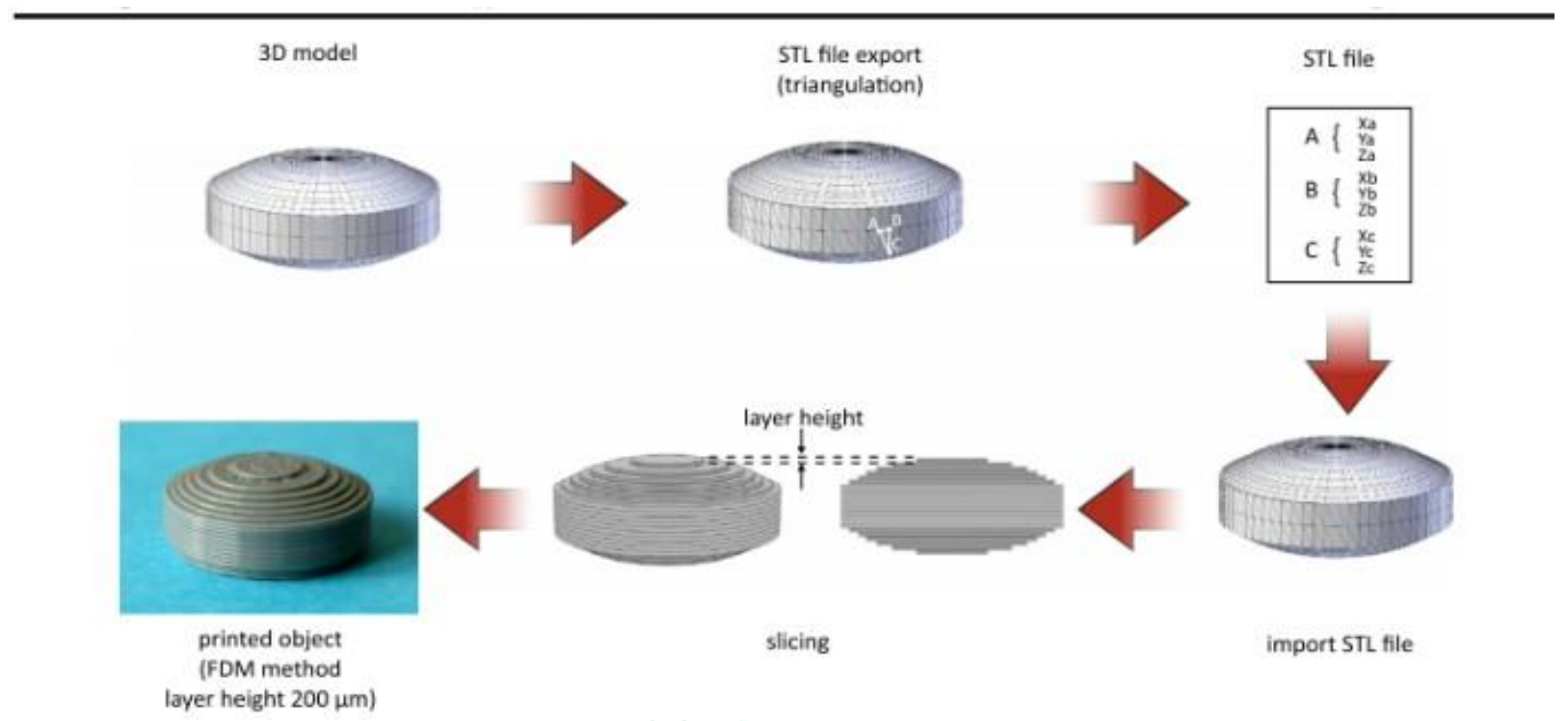

Figure 3: Fused Deposition Modeling printers

Fused Deposition Modeling printers use a thermoplastic filament, which is heated to its melting point and then extruded, layer by layer, to create a three-dimensional object. Each 3D printer which works according to a different mode requires enough material to be solidified and subsequent object fabrication. Despite of the diversity of 3D Printing methods, preparation of 3D-printed object includes several stages \& the design of 3D object with computeraided design software and optimization of the geometry according to printer specification, \& the export of 3D model to a common and printer recognizable file format e.g. Standard Triangle Language which includes only 3D geometry in form of each vertex position data or OBJ in which additionally information about polygonal faces or color texture are coded, $\&$ the import of the file to the software and generation of layers which will be printed; the height of the printed layer essentially influences the quality of the printed object as well as printing times \& the fabrication of the object by subsequent application (or solidification) of the material layers dedicated to the specific printing method. $[8]$

\section{Tele Pharmacy}

The Model State Pharmacy Act and Model Rules of the National Association of Boards of Pharmacy (Model Act) defines the practice of tele-pharmacy as "the provision of pharmacist care by registered pharmacies and pharmacists located within U.S. jurisdictions through the use of telecommunications or other technologies to patients or their agents at distances that are located within U.S. jurisdictions" and provides definitions of related terms (i.e., coordinating pharmacy, remote pharmacy, and remote dispensing site)1.Basically, Tele pharmacy is the use of telecommunications technology to provide pharmacist services to distant populations. Tele pharmacy services may include drug review/monitoring, provision of drug information, medication dispensing, verification of oral and sterile compounding, patient assessment, patient counseling and medication therapy management. The implementation of tele pharmacy systems is rapidly expanding due to the increasing affordability of videoconferencing technology and the increasing capability of the pharmacist to securely access electronic patient health records and perform traditional pharmacy practice activities remotely via the Internet. The number of scientifically rigorous evaluations of tele pharmacy has not kept pace with the rate of tele pharmacy adoption. [9]

Tele pharmacy undoubtedly is a great concept, but it is sometimes challenging to put into practice. Inherent to the adoption of these practices are legal challenges and pitfalls that need to be addressed. The start-up of tele pharmacy (hardware, software, connectivity, and operational cost) involves considerable time, effort, and money. For rural hospitals with fewer patients, the issue of costs appears to be one of the biggest barriers to tele pharmacy services. Moreover, execution and implementation of comprehensive and uniform tele pharmacy law is still a challenge. A welldeveloped system, however, can change the practice of pharmacy that is beneficial to both the rural communities and the hospitals or retail pharmacies that deliver these services. [10]

A literature review was conducted on basis of BIOMED CENTRAL and COCHRANE @ OVID databases. The keywords "Tele-Pharmacy", "e-Pharmacy" and "India" were used. The topics were selected to limit the citations with summaries, for the year between 2010 and 2014, in English. Almost twenty articles were used in this study. The word "TeleMedicine" was also added to figure articles relating TelePharmacy. Now, just ten articles not including "TelePharmacy" were found, which showed the lack of articles on the boundary Tele-Pharmacy and E-Pharmacy. [11] 


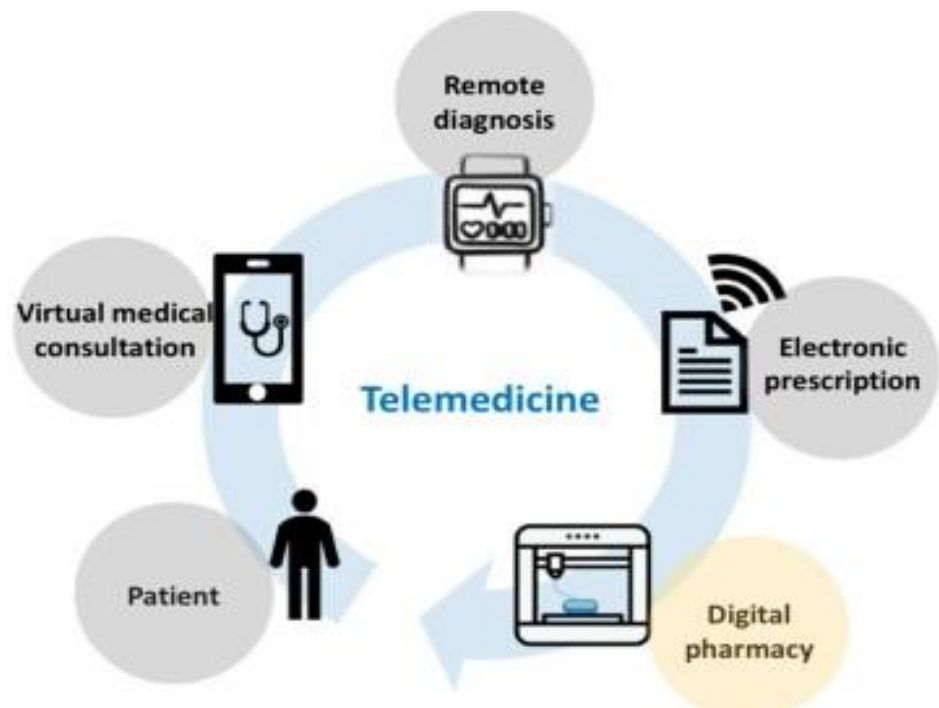

Figure 4: Tele pharmacy and its applications

- Traditional Pharmacy by virtual medical consultation

It diagnosed the provided signs and symptoms and gives a complete inventory of prescribed and nonprescribed drugs, including upfront solutions such as health and beauty tips, and beneficial items. [12]

\section{- $\quad$ Remote diagnosis Sites}

This model is used by a pharmacist at a central common pharmacy site who is providing a rural community a short distance away and who does not wish to manage two different prescription drug inventories at both the central pharmacy and remote site.

\section{- Electronic prescription by Hospital Tele pharmacy}

The hospital pharmacist has an approach to the patient's electronic medical records, and checks the prescription for proper dosing, allergies, imitation of drug therapy and drug interactions. Then the pharmacist electronically approves the dispensing of the prescription through a specialized electronic device.

\section{- Automated Dispensing Machines (Digital pharmacy)}

The prescriber's drug order is provided to the licensed pharmacist at a central pharmacy site electronically or by fax. The licensed pharmacist go through the patient's profile, do the proper effective use of drug review and then commands the dispensing machine to release the medication. The patient is then advised, by the pharmacist, through the computer by audially or visually. [13]

\section{Personalized medicines}

Personalized Medicines are activity of medical treatment to the person's unique attribute. They are very rangy snappy and liberal techniques in the pharmaceutical field that is informed by every individual's genetical property and surrounding which they are receptive to beyond doubt diseases. [13] At present, personalized medication is mainly applied in the following areas for prediction, diagnostics, therapeutics (including outcome evaluation) and drug development. [14] Also, they have a great impact on customized drug delivery system. Personal genomics connect genotype to phenotype and provide insight into disease. Pharmacogenomics connect genotype to patient specified treatment. [14]

It helps identify the best treatment for an individual, thus effectively tailoring and customizing treatment for each one by their gene's information. In many diseases, precision medicine depends on molecular biomarkers, molecular events that relate to response of the treatment and clinical outcome but not necessarily cautious to the disease. Currently, almost all programs of precision medicine depend on patient's DNA sample examination, range from highly focused to specific regions (a few genes are sequenced), to whole-exome (all genes are sequenced) and whole-genome (the entire genome is sequenced). [15]

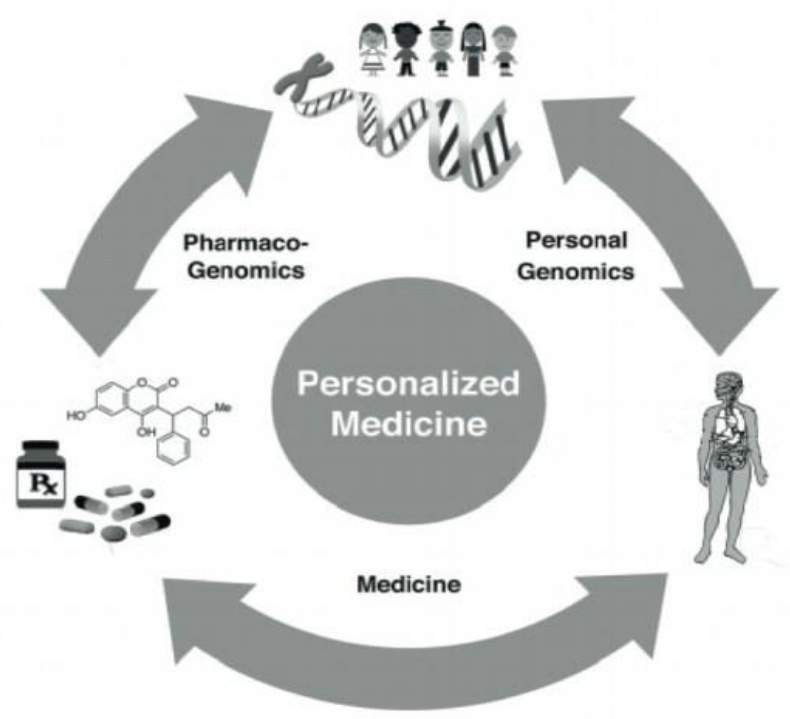

Figure 5: Personalized medicine

Personalized medicine can be prominent in the medicine from reaction to anticipation. [16] Personalized medicine can supervise the selection of most favorable therapy and minimize trial-and-error prescribing. It can avoid or at least reduce adverse drug reactions. It surely will prove to have increased patient compliance and hence the quality of life. Moreover, it can have a control on the health care cost. [17] 


\section{Bioelectronics Medicines}

Bioelectronic Medicine (BEM) can change radically improve medicine, therapy and brilliantly improve the consequences of healthcare. It makes use of electrical, magnetic, optical, ultrasound, etc. pulses to affect and alter neurological behavior which in turn impacts body tasks as an alternative to drug-based involvements. Further, it provides the chances for targeted and personalized treatments of neurological based diseases and conditions in closed-loop control systems. [18]

Bioelectronic medicine is a new appeal for treating major chronic diseases that could give doctors and patients' alternatives to costly used medicine and may become as commonly prescribed as chemical or biological drugs. Some researchers and pharmaceutical companies are already taking this potential new class of treatments seriously and due to promising results, others are expected to follow. [19]
Imagine a prescription from your doctor, not for tablets but for a tiny electrical device implanted on a nerve in your neck. The device will monitor and treat your condition - whether it is diabetes, asthma, hypertension or even cancer - by modulating electrical impulses. [20]

In clinical trials, they have been increasingly applied technology and gaining more popularity. Some devices used in curing include cochlear and retinal implants and vagus nerve stimulators. However, all cells can be caused by electrical inputs which give rise to the chance to broaden the use of bioelectronic medicine for treating disease. Electronic actuation of non-excitable cells has been shown to lead to 'programmed' cell behavior via application of electronic input which alters key biological processes. [21]

Bioelectronic medicines have a great impact on neuromodulation. Figure 6 shows how this technique has a hand in treating rheumatoid arthritis.

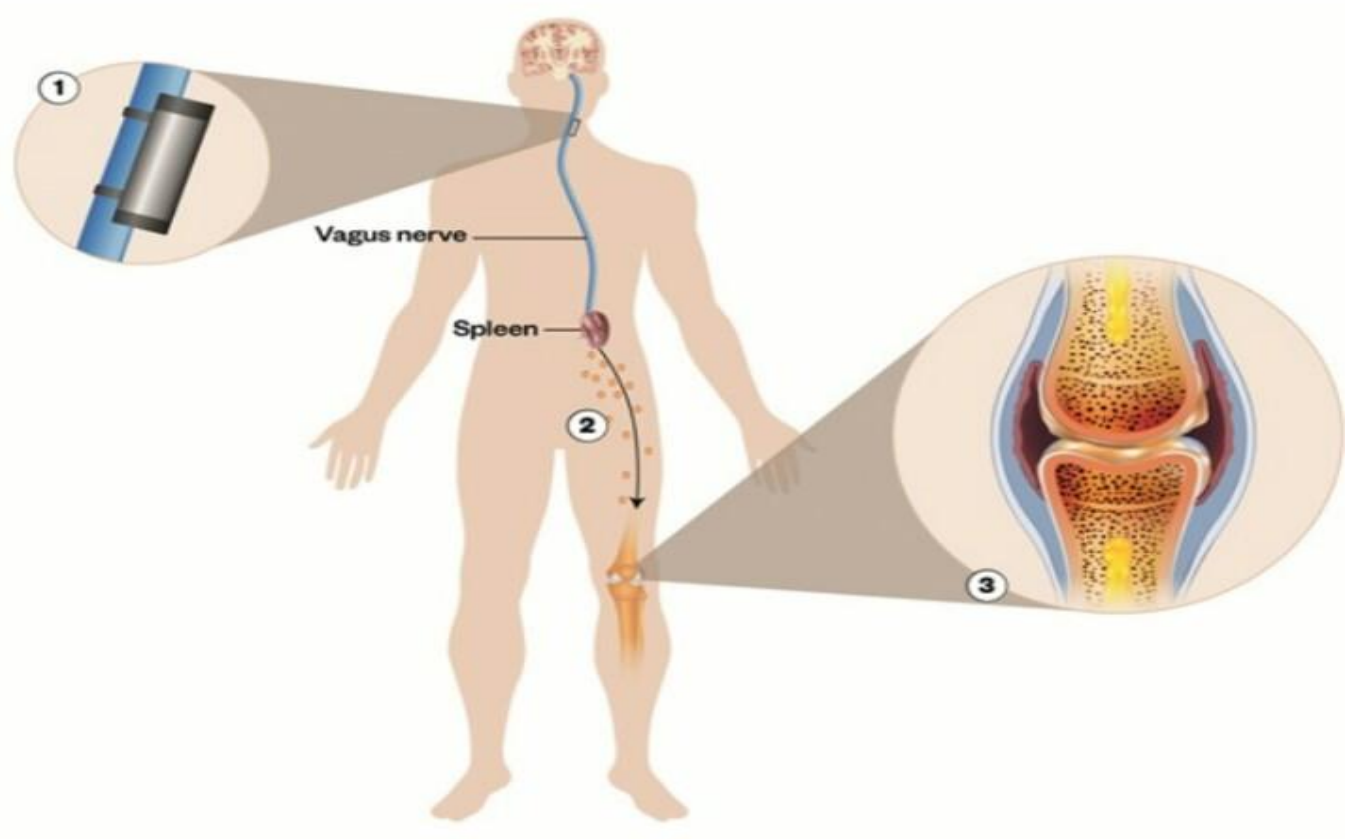

Figure 6: Treatment through bioelectronic devices

Vagus nerve produces the electrical stimulation with a bioelectronic device affects splenic T-cells and macrophages to decrease production of tumour necrosis factor (TNF), an inflammatory mediator, leading to decreased rheumatoid arthritis symptoms in some patients. The mode of action of the device is as follows. [22]

1) A microregulator energizes the vagus nerve. The signals go through the vagus and splenic nerve trigger depletion in the activation of $\mathrm{T}$ cells and macrophages in the spleen.

2) There is a reduced production of systemic inflammation mediators and reduced activation of circulating immune cells.

3) This leads to reduced inflammation, joint damage and pain.

\section{Conclusion}

The article describes few recent developments in technology like artificial intelligence, personalized medicines, customized medicines, 3D printing, bioelectronic devices and tele pharmacy, which have the potential to augment health care and drug delivery in coming times. Today because of the advancement of science man is taking help of artificial intelligence in the various operations of pharmaceutical industries like automation and robotics in a range of pharmaceuticals, chemical analysis and the developments in dissolution testing and the analytics processing of metereddose inhalers etc. The era of one pill fits all is also going to finish as scientists are developing personalized and customized drug delivery system based on individual patient genetic build up. Pharmacogenetics will be the future of treatment in the healthcare arena.

In the area personalized medication one of technologies which is achieving major developments is the 3D printing. The possibility of 3D printing for developing dosage forms like tablets, capsules to the production of medical devices parts can have a huge impact on the way medical care industry functions. Bioelectronic devices and medicines will be able to modulate neural signaling patterns and hence achieve therapeutics effects. Tele pharmacy is basically the 
use of telecommunications technology that enables pharmacists to provide services to the patients who are stationed at distant places. In a nutshell all these advancements can improve the therapy methods and enhance the patient care to a great extent.

\section{References}

1. Dasta JF. Application of artificial intelligence to pharmacy and medicine. Hosp Pharm 1992; 27:312-5,319-22.

2. Manikiran,, S. S., and Prasanthi, N. L. Artificial Intelligence: Milestones and Role in Pharma and Healthcare Sector. Pharma times 2019; 51:9-56.

3. Warwick, Kevin \& Coleman, Kenneth \& Leiper, K. \& Crook, M. \& Andrew, R. \& Parsley, K. \& Plummer, G. \& Clark, D.. Robotics and automation in the pharmaceutical laboratory. 1997; 19:51-54.

4. Ibrić, s. V. E. T. L. A. N. A., parojčić, j. E. L. E. N. A., petrović, j. E. L. E. N. A., and djurić, z. O. R. I. C. A. artificial intelligence in pharmaceutical product formulation: neural computing*. Chemical Industry \& Chemical Engineering Quarterly 2009; 25:1-10.

5. Jose, P. A., and Christoper, P. 3D printing of pharmaceuticals a potential technology in developing personalized medicine. Asian Journal of Pharmaceutical Research and Development 6, $46-54$.

6. Scoutaris, N., ROSS, S. A., and Douroumis, D. 3D printed "Starmix" drug loaded dosage forms for paediatric applications, 2-19

7. Horst, Diogo. 3D Printing of Pharmaceutical Drug Delivery Systems. Archives of Organic and Inorganic Chemical Sciences. 2018; 1:1-5. 10.32474/AOICS.2018.01.000109.

8. Araújo, M. R. P., Sa-Barreto,, L. L., Gratieri,, T., Gelfuso, G. M., and Cunha-Filho, M. The Digital Pharmacies Era: How 3D Printing Technology Using Fused Deposition Modeling Can Become a Reality. Pharmaceutics 2019; 1-14.

9. Rajiah, Kingston \& Venkataraman, R. Tele-pharmacy and Epharmacy equivocal in India? Technological and legal issues of tele-pharmacy in India. International Journal of Current Pharmaceutical Review and Research. 2015; 6:89-93.

10. Peterson,, C. D., Anderson,, H. C., Bettesworth, L., and Lockyear M. Telepharmacy. chapter 10 ( Lordan,, D., Davies,, J., and Constable, J., Eds.) 1-35.
11. Skrei, A., and Rundquist, M. M. Pharmacy Services in Telepharmacy: how 's it working, where it' s working, and what's required to practice in this new setting. Advances in Pharmacy: Journal of Student Solutions to Pharmacy Challenges is published by the University of Minnesota Libraries Publishing. 2017; 1:1-15.

12. Poudel, A., and Nissen, L. M. Telepharmacy: a pharmacist's perspective on the clinical benefits and challenges. Integrated pharmacy reasearch and practiCE 2016; 1-8.

13. Marlise Araújo dos Santos1,4, Patrícia Bellicanta Lazzarotto1,4, Helena Willhelm de

Oliveira2,4, Júlio César M de Lima3,4 and Thais Russomano, Telepharmacy: The Pharmacy of the 21st Century. SM GROUP 2017; $1-5$.

14. Meiliana, A., Dewi, N. M., and Wijaya, A. Personalized Medicine: The Future of Health Care. The Indonesian Biomedical Journal 2016; 8:127-146.

15. Meyer UA.The Potential limits of Personalized medicine: a personal view. Clin Pharmacol Ther. 2012; 373-5.

16. Chouchane, Lotfi \& Mamtani, Ravinder \& Dallol, Ashraf \& Sheikh, Javaid. Personalized Medicine: A Patient - Centered Paradigm. Journal of translational medicine. 2011; 9:206. 10.1186/1479-5876-9-206.

17. Schooneveldt, B. C., Veldwijk, J., and Weda, M. Application of personalized medicine. Ministry of health, welfare and sport 2015; 3-90.

18. Dorey, E. (2016) Acting on the potential of action potentials: will bioelectronic medicines be the next biologics? The Pharmaceutical Journal 1-11.

19. Pavlov, V. A., and Tracey, K. J. Bioelectronic medicine: updates, challenges and paths forward. Pavlov and Tracey Bioelectronic Medicine 2019; 5:1, 1-4.

20. Gordonov T, Kim E, Cheng Y, Ben-Yoav H, Ghodssi R, Rubloff G, Yin JJ, Payne GF, Bentley WE:Electronic modulation of biochemical signal generation. Nat Nanotechnol 2014; 601610.

21. Olofsson, P. S., and Tracey, K. J. Bioelectronic medicine: technology targeting molecular mechanisms for therapy. The Association for the Publication of the Journal of Internal Medicine Journal of Internal Medicine. 2017.

22. Birmingham, Karen \& Gradinaru, Viviana \& Anikeeva, Polina \& Grill, Warren \& Pikov, Victor \& McLaughlin, Bryan \& Pasricha, Pankaj \& Weber, Douglas \& Ludwig, Kip \& Famm, Kristoffer. Bioelectronic Medicines: A Research Roadmap. Nature Reviews Drug Discovery. 2014; 13. 399. 10.1038/nrd4355. 